\title{
Reducing Complexity of FIR Filters using Narrowband Filters
}

\author{
P.U. Bilgunde \\ MBES College of Engineering, \\ Ambajogai
}

\author{
S.K. Sudhansu \\ MBES College of Engineering, \\ Ambajogai
}

\author{
S. M Jagde, Ph.D \\ College of Engineering \\ Osmanabad.
}

\begin{abstract}
Reducing complexity of FIR filters using narrowband filters presents a practically efficient Narrowband FIR Filter designed by using Frequency Response Masking Technique \& the Interpolated FIR technique. The Proposed method cascades the interpolated filter with masking filter. By giving the filter design coefficients (filter characteristics) we can determine the filters interpolated magnitude coefficients. \& then this is given to Narrowband Filter will get the impulse response with less number of taps. At the last step we can remove the unwanted part by using the masking filter.
\end{abstract}

LabVIEW software is used to implement the reduced complexity FIR filter using narrowband filter. It was shown that the resulting filter is practically efficient and also the errors in human Audiogram are minimized.

\section{General Terms}

Sampling Frequency, Ripple Factor, Attenuation, Pass-band, Stop-band.

\section{Keywords}

Narrowband FIR Filter, Frequency Response Masking Technique, Interpolated FIR Technique

\section{INTRODUCTION}

The order of FIR filter is relatively inversely proportional to the transition bandwidth [1].

Number of Taps $\mathrm{T}_{\mathrm{P}}=\mathrm{f}_{\mathrm{s}} / \Delta \mathrm{f}$

fs $=2 *$ fmax ; if $\mathrm{N}$ is odd

$$
=2 * \mathrm{fmax}+\Delta \mathrm{f} ; \text { if } \mathrm{N} \text { is even }
$$

Where " $f s$ " is sampling frequency \& " $\Delta f$ " is Transition Bandwidth [1].Conventional FIR filters with narrow transition bands and high orders might be too complex to implement. We might consider designing narrowband filters using the IIR filters. However narrowband IIR filters typically have nonlinear phase especially near the transition band and are numerically sensitive.

Conventional FIR filters with narrow transition bands and high orders might be too complex to implement. We might consider designing narrowband filters using the IIR filters. However narrowband IIR filters typically have nonlinear phase especially near the transition band and are numerically sensitive, therefore the narrowband FIR filter is used.

While modeling Human Audiogram of arbitrary magnitude characteristics, 11-samples 8-samples \& 6-samples are considered in the range 125 to $8 \mathrm{kHz}$. If we increase the number of samples then we will get the magnitude errors between designed FIR filter \& Human Audiograms are very less [16]. That means by increasing number of samples the designed FIR filter can be matched with Human Audiogram. But that becomes practically inefficient as number of taps required for designing the filter also increases and due to this the cost \& complexity of filter increases.

Thus to reduce complexity without increasing magnitude errors, the DFD Narrowband Filter Design Virtual Instrument is used. This VI uses the interpolated FIR techniques \& Frequency Response Masking Technique to design narrowband FIR filters with significantly less computational complexity than conventional FIR solutions.

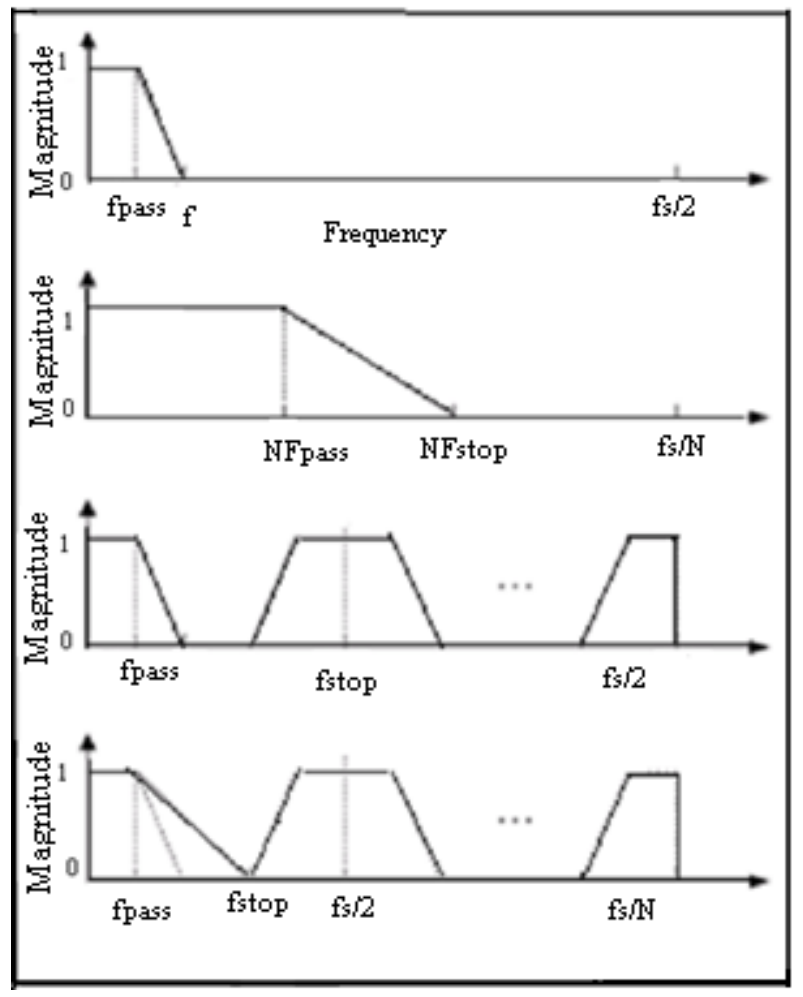

Fig.1 Frequency Response of Low Complexity Filters[14]

The next section reviews the Interpolated FIR technique [14]. In section 3 Frequency Response Masking techniques [12] has explained. The proposed method is described in section 4 . Section 5 explains the LabVIEW implementation of the proposed method. Section 6 describes the comparison between conventional filter and narrowband filter. The results are shown in section 7. Section 8 presents the conclusion of the paper.

\section{INTERPOLATED FIR FILTER COEFFICIENT TECHNIQUE}

The interpolated FIR filter coefficients are constructed by inserting $\mathrm{N}-1$ zeros between adjacent coefficients of model filter. The model filter has a wider transition band than the 
target narrowband filter and determines the spectrum shape of the target narrowband filter [14].

The Frequency response of low complexity filters is as shown in Fig.1

\section{FREQUENCY RESPONSE MASKING TECHNIQUE}

To remove the unwanted images at higher frequencies the FRM filter is need to be cascaded to IFIR Filter [14]. A Masking filter is designed in such a way that it is able to remove the pass band at higher frequencies. For the human audiogram the pass band is at $125 \mathrm{~Hz}$ to $8 \mathrm{KHz}$, so the pass band at higher frequencies are removed.

\section{PROPOSED METHOD}

Figure 2 shows the diagram of proposed method. The first step is to take in the filter characteristics i.e. sampling frequency, ripple, attenuation, pass-band, stop-band of the required filter.

The second step is to determine the interpolated magnitude coefficients by using the filter characteristics which is input to the Interpolated FIR VI. Here in the interpolated filter coefficients we get the model filter and image suppressor coefficients.

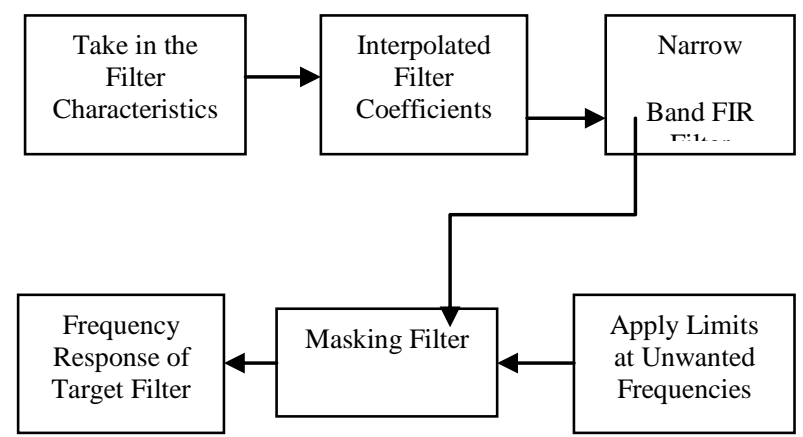

Fig.2 Proposed Method of Target Narrowband Filter

In the third step the narrowband FIR filter is designed using the interpolated filter coefficients. And at the output the frequency response of narrowband filter can be obtained.

In the last step we can apply the appropriate limits to the frequency response of narrowband filter by using masking filter and the required frequency response is obtained.

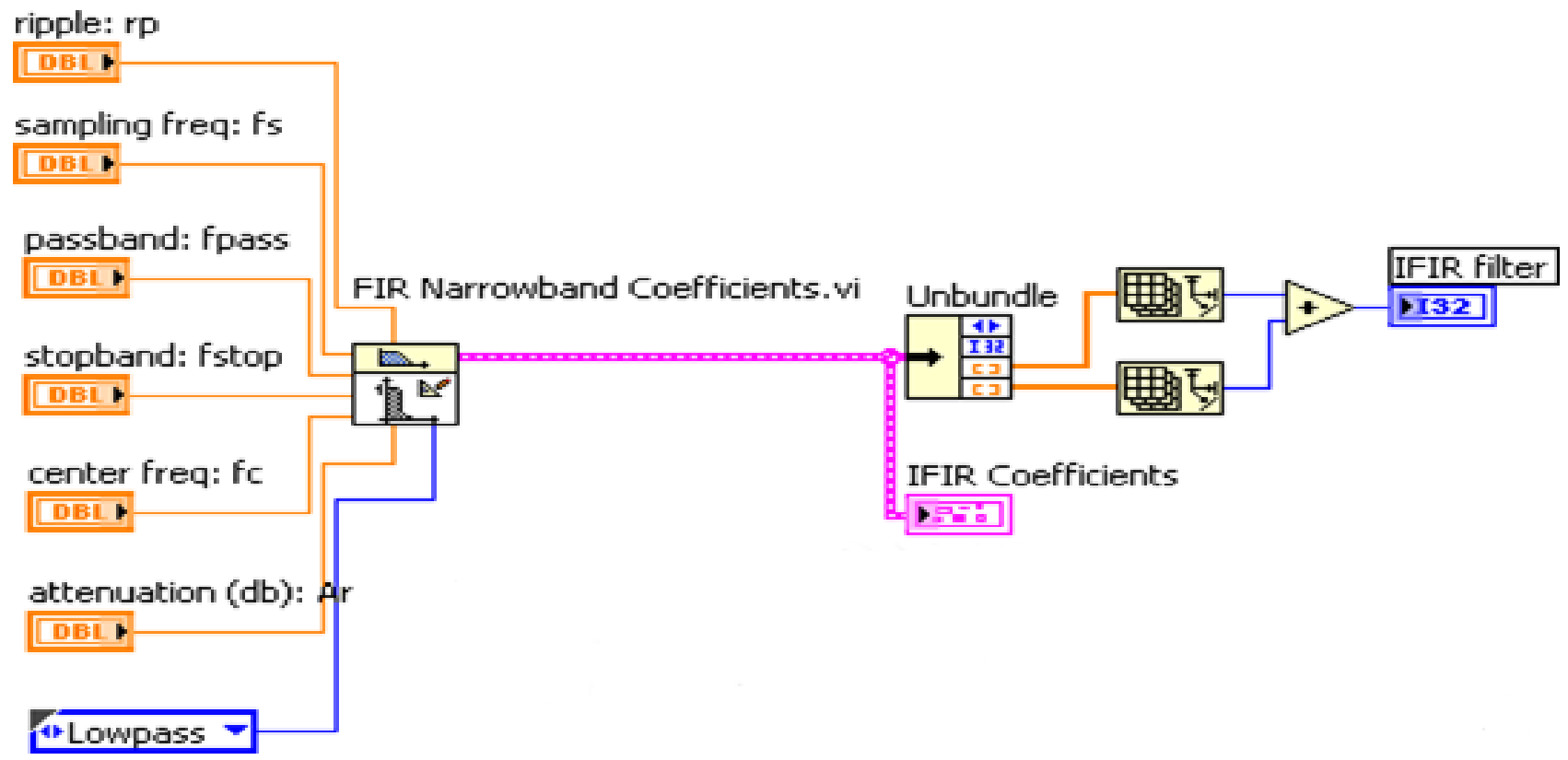

Fig.3 Interpolated FIR Filter

\section{LABVIEW IMPLEMENTATION OF THE FILTERS}

The virtual instrument (VI) to generate the interpolated filter coefficients is shown in figure 3. Here the IFIR tool is used to generate the interpolated filter coefficients. The filter characteristics i.e. pass band, stop band, attenuation, ripple factor are needed to get the required interpolated filter coefficients. At the output the corresponding frequency response is compressed $\mathrm{N}$ times called as model filter. Where
$\mathrm{N}$ is interpolation factor. By using the model filter \& image suppressor coefficients the size of interpolated filter can be obtained from the following formula

Size of IFIR filter $=$ Size of model filter + Size of image suppressor

The next step is to filter out the interpolated frequencies; here we use the narrowband FIR filter. The VI to implement the narrowband FIR filter is shown in Fig.4. The N-1 zeros are padded between two adjacent coefficients of model filter. 


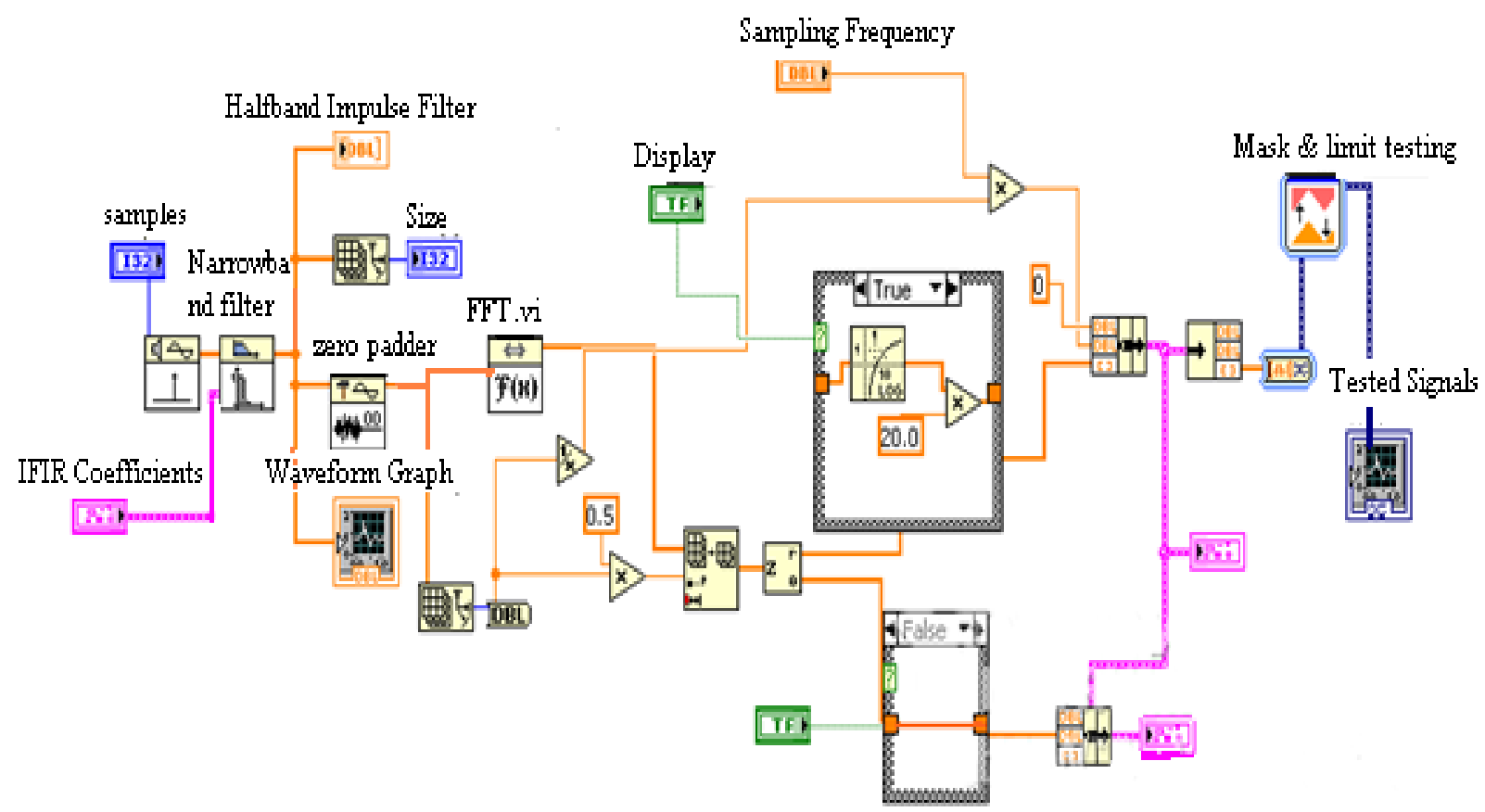

Fig.4 DFD Narrowband Filter Design VI

Here the frequency response is $\mathrm{N}$ times narrower and the $\mathrm{N}-1$ image frequency responses are produced (unwanted spectrum). At the output we get the frequency response of narrowband FIR filter but it contains the frequency response at higher frequency also.

Therefore the next step is required i.e. Masking Filter. The masking filter is used to remove the unwanted images at higher frequencies and low frequencies both. The VI to implement masking filter is shown in Fig.4.

In this paper we are analyzing only the human audiogram, so we require frequency between $125 \mathrm{~Hz}$ to $8 \mathrm{KHz}$. Therefore it is needed to design the filter by giving appropriate limits. At the output we get the required frequency response only. And the response at higher frequencies is masked.

\section{COMPARISON OF NARROWBAND FILTER AND CONVENTIONAL FIR \\ FILTER}

To implement the FIR filter that matches the human audiogram, we require the FIR filter with increased number of samples. The conventional FIR filter with increased number of samples has very less magnitude errors as compared to human audiogram; i.e. completely matches the response of human audiogram. But as we increase number of samples the coefficients and also the number of taps of filter increases. As a result of this hardware \& complexity of filter increases and that becomes practically inefficient to implement.

If Narrowband FIR Filter is considered for modeling human audiogram, the required response can be obtained at lower cost and complexity. Because here Narrowband filter design VI that uses interpolated FIR techniques and Frequency As shown in Table 1. the conventional method requires more number of taps than the interpolation method. Therefore the hardware cost and complexity of the filter is reduced. And response masking technique is used to design narrowband filter.

\section{RESULTS}

The magnitude response of the narrowband FIR filter is shown in Figure 6. Here we get the very less magnitude errors of resulting filter as compares to human audiogram. That means the designed filter matches the human audiogram with less computational complexity.

The frequency response of masking filter is shown in Figure 7. In this figure the response of narrowband filter can be obtained as per the requirement. Here the upper and lower limits are applied and the higher frequencies are masked $\&$ the lower frequencies are passed.

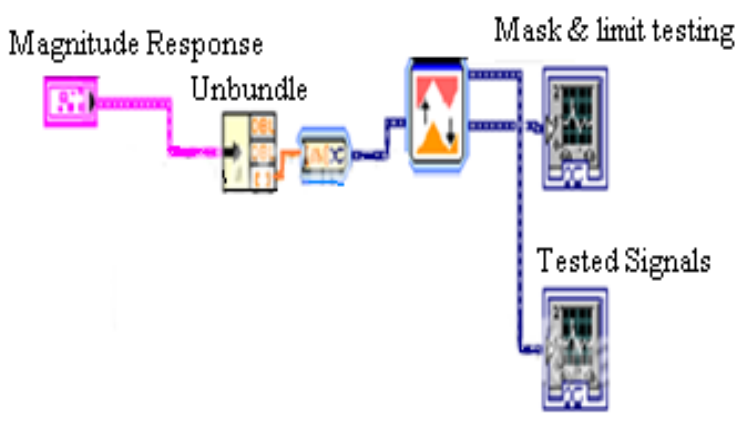

Fig.5 Masking Filter

also the magnitude errors and spread of filter coefficients are also reduced. 


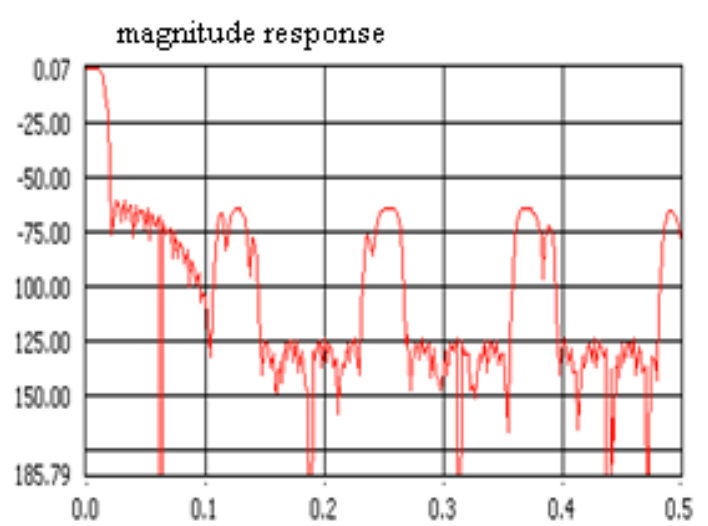

Figure 6.Magnitude Response of Narrowband FIR Filter

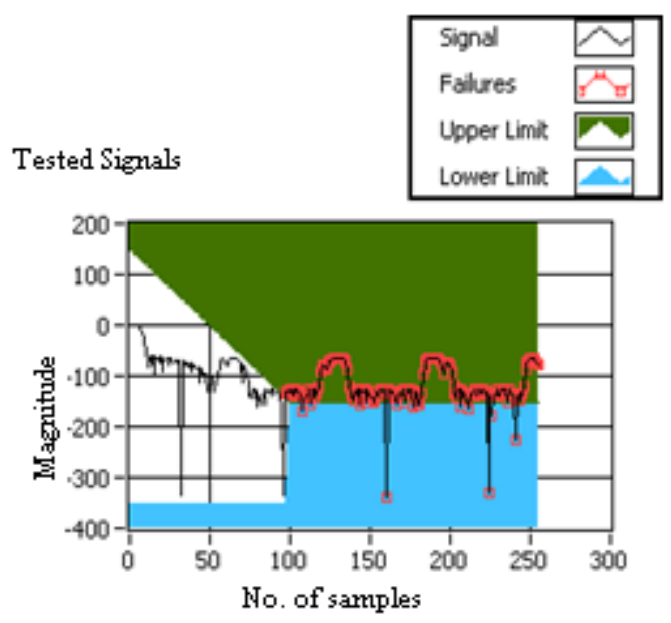

Fig. 7 Tested Signals Using Masking Filter

Table 1. Taps required for different number of samples

\begin{tabular}{|c|c|c|}
\hline $\begin{array}{c}\text { Number } \\
\text { of } \\
\text { Samples }\end{array}$ & $\begin{array}{c}\text { Number Of Taps by } \\
\text { Conventional } \\
\text { method }\end{array}$ & $\begin{array}{c}\text { Number of Taps by } \\
\text { Interpolation method }\end{array}$ \\
\hline $\begin{array}{c}20 \\
\text { samples }\end{array}$ & 74 & 26 \\
\hline $\begin{array}{c}15 \\
\text { samples }\end{array}$ & 57 & 21 \\
\hline $\begin{array}{c}11 \\
\text { samples }\end{array}$ & 41 & 17 \\
\hline 8 \\
samples
\end{tabular}

\section{CONCLUSION}

This paper presents a practically efficient Narrowband FIR Filter that analyses the human audiogram. The Proposed method uses the narrowband filter cascaded to the masking filter. Narrowband filter uses the interpolated FIR technique. Therefore we can analyze large band of frequencies and then the output response of narrowband FIR filter is masked using the masking filter as per requirement.
The human audiogram designed using conventional filter has some magnitude errors of matching. These errors can be minimized by increasing number of samples, but that becomes practically inefficient. Here it has designed the filter with reduced number of taps. Therefore the magnitude errors are minimized without increasing hardware cost and complexity of filter.

\section{ACKNOWLEDGEMENTS}

Our special thanks to experts who have guided towards development of this paper.

\section{REFERENCES}

[1] James T. George, Elizabeth Elias "Continuously Variable Bandwidth Sharp FIR Filters with Low Complexity," Journal of Signal and Information Processing, 2012, 3, 308-315

[2] E. Szopos, M. Neag, I. Saracut, H. Hedesiu, L. Festila "A Method for Designing FIR Filters with Arbitrary Magnitude Characteristic Used for Modeling Human Audiogram," Advances in Electrical and Computer Engineering Volume 12, Number 2, 2012.

[3] T. W. Parks ,C. S. Burrus, "Digital filter design", John Wiley \& Sons Inc.,pp. 33-44,1987.

[4] E. Szopos, M. Topa, L. Festila, H. Hedesiu, "FIR synthesis of the human hearing mechanism response", Acta Technica Napocensis, Electronics and Telecommunications,pp. 41-44,2010.

[5] E. Szopos, H. Hedesiu, "LabVIEW FPGA based noise cancelling using the LMS adaptive algorithm", Acta Technica Napocensis, Electronics and Telecommunications,pp. 41-44,2010.

[6] Y. Lian, Y. Wei, "A computationally efficient nonuniform FIR digital filter bank for hearing aids", IEEE Trans. on Circuits and Systems, 2005. [Online]. Available:http://ieeexplore.ieee.org/stamp/ stamp.jsp?tp=\& arnumber $=1556782 \&$ isnumber $=33114$.

[7] J. Huopaniemi, M. Karjalainen, "HRTF filter design based on auditory criteria", Proc. Nordic Acoustical Meeting, 1996.

[8] Dr. Yong Lian "The Design of High Speed Low Power Digital FIR Filters Based on Frequency-Response Masking Technique," IEEE CAS Workshop, 2 March 2007, Vancouver.

[9] Y.C. Lim and Y. Lian, "Frequency-response masking approach for digital filter design: complexity reduction via masking filter factorization," IEEE Transactions on Circuits and Systems II, vol. CAS-II-41, no. 8, pp. 518525, August 1994.

[10] Y. C. Lim1, Y. J. Yu1, H.Q. Zheng2 and S.W. Foo1 FPGA Implementation Of Digital Filters Synthesized Using The Frequency-Response Masking Technique

[11] Yong Ching Lim, Member, IEEE "Frequency-Response Masking Approach for the Synthesis of Sharp Linear Phase Digital Filters," IEEE TRANSACTIONS ON CIRCUITS AND SYSTEMSV,O L. CAS-33, NO. 4, APRIL 1986.

[12] O. O. Khalifa, M. H. Makhtar, M. S. Baharom, "Hearing aids system for impaired people", International Journal of Computing \& Information Sciences, 2004. 
[13] NI Tutorial "Narrowband FIR Filters, Digital Filter Design Toolkit, LabVIEW 2011.

[14] G. Jovanovic and A. Sarmiento, An Efficient Method for Narrowband FIR Filter Design,"Computacion y Sistemas Vol.2 Nos. 2-3 pp.78-86 @1999, CIC - IPN. ISSN 14055546 Impreso en Mexico.
[15] Syed Asad Alam and Oscar Gustafsson"Implementation of Narrow-Band Frequency Response Masking for Efficient Narrow Transition Band FIR Filters on FPGAs", 978-1-4577-05 16-8/11/\$26.00 @2011 IEEE.

[16] A. Antoniou, "Digital Signal Processing", McGraw Hill ,pp 425-458,2005. 